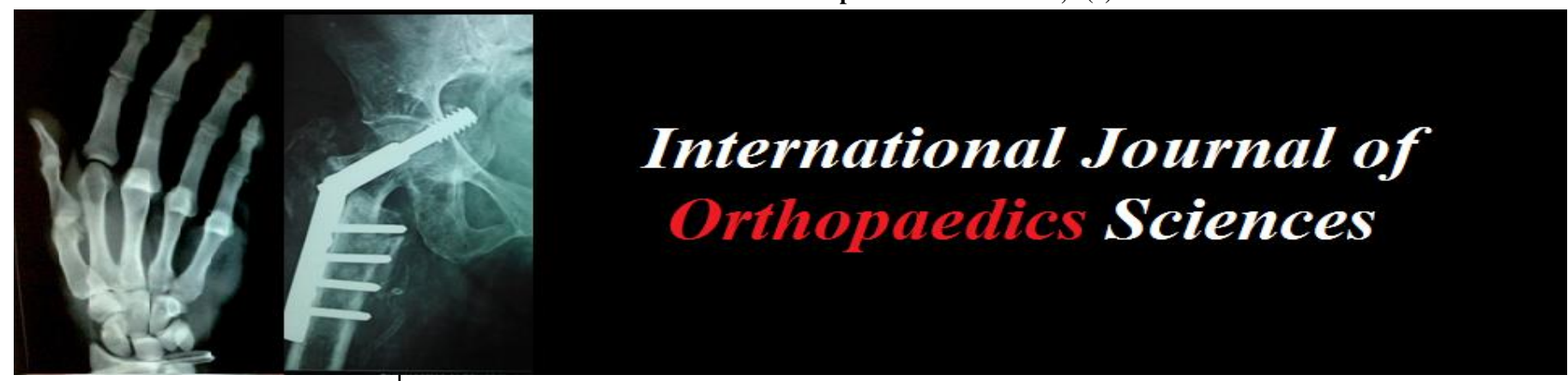

ISSN: $2395-1958$

IJOS 2019; 5(2): 133-137

(C) 2019 IJOS

www.orthopaper.com

Received: 09-02-2018

Accepted: 12-03-2018

Dr. Gutha Sethu Kumar Registrar, Department of Orthopaedics, Manipal Hospital, Bangalore, Karnataka, India

Dr. Ajay Kumar SP

Registrar, Department of

Orthopaedics, Manipal Hospital

Bangalore, Karnataka, India

Dr. Sanjay Prasad Hegde Consultant, Department of Orthopaedics, Narayana Hrudayalaya, Bangalore, Karnataka, India

Dr. Samarth Arya

Fellow in Hip and Knee,

Department of Orthopaedics, Wrightington Hospital, United Kingdom

Dr. Anoop Pothen John Associate Consultant, Department of Orthopaedics, Manipal Hospital, Bangalore, Karnataka, India

Dr. Pradeep Jadhav

Junior resident, Department of Orthopaedics, Manipal Hospital, Bangalore, Karnataka, India

Correspondence

Dr. Samarth Arya

Fellow in Hip and Knee,

Department of Orthopaedics,

Wrightington Hospital, United

Kingdom

\section{A clinical study to evaluate the effectiveness of proximal femoral nail antirotation in the management of peritrochanteric fractures of the femur}

\author{
Dr. Gutha Sethu Kumar, Dr. Ajay Kumar SP, Dr. Sanjay Prasad Hegde, \\ Dr. Samarth Arya, Dr. Anoop Pothen John and Dr. Pradeep Jadhav
}

DOI: https://doi.org/10.22271/ortho.2019.v5.i2c.25

\section{Abstract}

Background: Fractures around the trochanteric region of the femur are one of the commonest fractures encountered in orthopedics in elderly and young patients. In younger patients, the fractures usually result from high-energy trauma like RTA or fall from height and older patients suffering from a minor fall can sustain fracture in this area because of weakened bone due to osteoporosis.

Methods: Forty-nine patients, aged above 40 years with closed intertrochanteric fractures who were operated using proximal femoral nail anti-rotation (PFN-A) were evaluated for functional outcome and analyzed for the technical problems and complications associated with surgery.

Results: Nineteen patients were less than 60 years of age group and all patients of this group had excellent and good outcome. 25 patients who belonged to more than 60 years of age group of which 7 patients had fair and poor outcome and it was statistically significant $(\mathrm{p}=0.0139)$.

Conclusion: Closed reduction and internal fixation using PFN-A is an excellent method of treatment for an intertrochanteric fracture, to provide rapid recovery and good functional outcome. Age is a significant predictor of final outcome following surgical treatment. Younger is the age better the outcome.

Keywords: Intertrochanteric Fractures, Proximal femoral nail anti-rotation (PFN-A), Closed reduction and internal fixation

\section{Introduction}

Fractures around the trochanteric region of the femur are one of the commonest fractures encountered in orthopedics and also the most devastating injuries of the elderly. The incidence of this fracture increases with advancing age. Growing number of population and the road traffic accidents have resulted in an enormous increase in these type of fractures ${ }^{[1,2]}$. In younger patients, the fractures usually result from high-energy trauma like RTA and fall from height and accounts for only $10 \%$ percent. Older patients suffering from a minor fall can sustain fracture in this area because of weakened bone due to osteoporosis or pathological fracture and this account for $90 \%{ }^{[3]}$.

\section{Methodology}

The purpose of this prospective study conducted in Department of Orthopedics, Manipal Hospital, Bangalore was to evaluate the functional outcome of proximal femoral nail antirotation (PFN-A) for management of intertrochanteric fractures and to analyze the technical problems and complications associated with surgery.

Forty-nine patients, aged above 40 years with closed intertrochanteric fractures who were operated between June 2014 to May 2015 were included in the study. Patient with delayed presentation, congenital or acquired deformities of hip, associated with fractures of acetabulum, pubis, extending to femur shaft on same side, pathological fractures were excluded from the study.

On admission of the patient, a careful history was elicited from the patient and/or attendants to reveal the mechanism of injury and the severity of trauma. The patients were then assessed 
clinically to evaluate their general condition and a complete survey was done to rule out significant injuries. Careful examination was done to rule out fractures at other sites.

Follow up of cases was done at regular intervals of 4 weeks for minimum of 6 months and then after once in 3 months till one year. Patient was assessed clinically regarding hip and knee function, walking ability, fracture union, deformity and shortening. At the end of one year, Harris hip score was measured and X-ray of the involved hip with femur was done to assess fracture union and implant bone interaction.

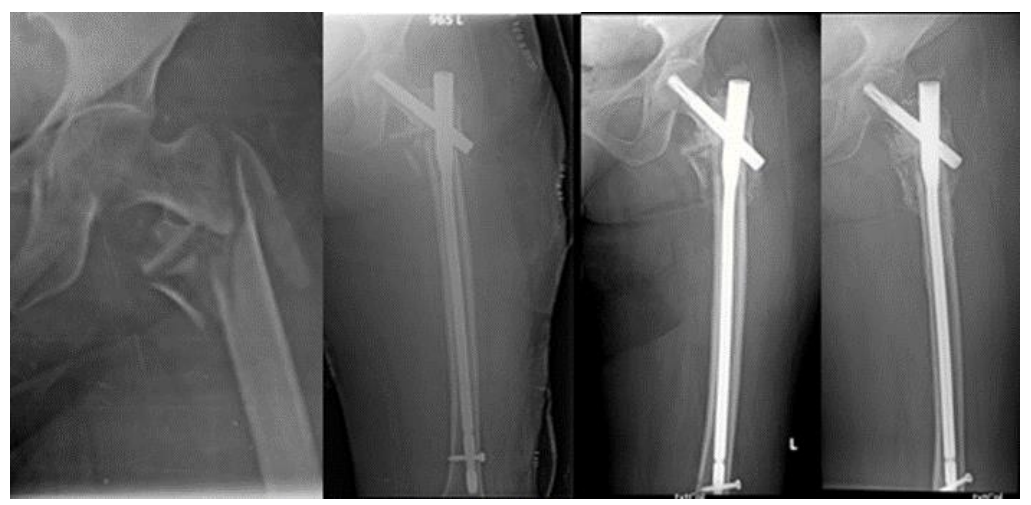

Fig 1: X-ray Preoperative and Immediate Postoperative, at 7 and 16 weeks

\section{Observation and Results}

Forty-Nine cases of peritrochanteric fractures treated by proximal femoral nail antirotation (PFNA). Three patients were lost follow-up and 2 patients died at the end of one year, so 44 cases were available for final study. The following were the observations made and the available data is analyzed as follows.

In this study, 19 patients were less than 60 years of age, 19 patients were in the age group between 61-80 years and 6 patients were more than 80 years of age group. Mean age of the study population was 65.02 years. There were $25(57 \%)$ male and $19(43 \%)$ female patients in our study.

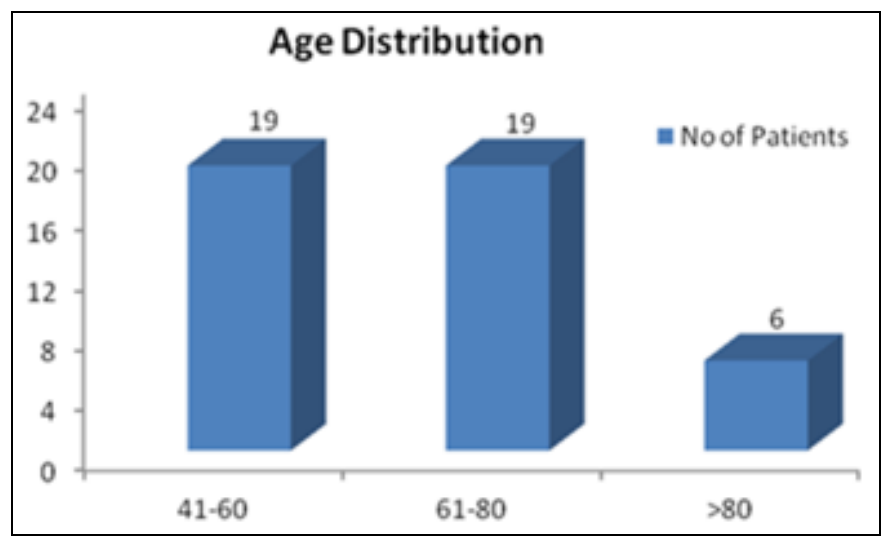

Graph 1: Age distribution

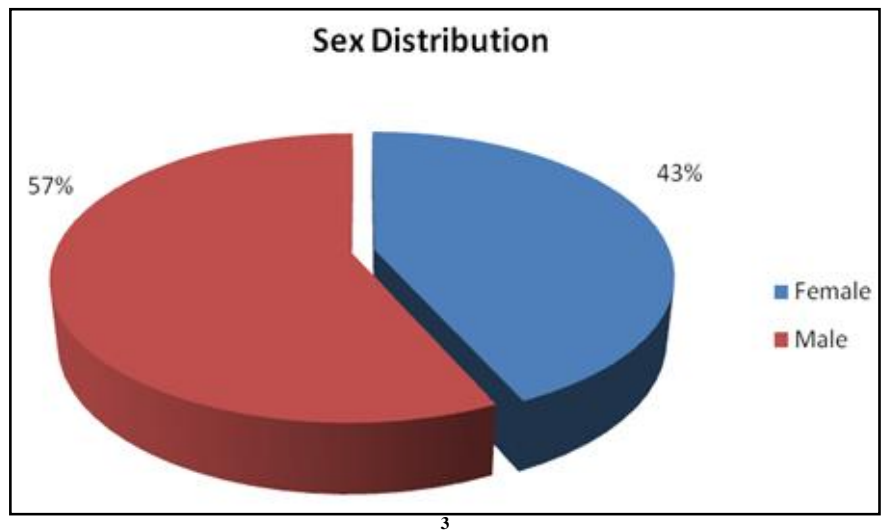

Graph 2: Sex distribution
There were $23(52 \%)$ patients having type II fracture, 11 (25\%) patients with type I fracture and $10(23 \%)$ patients with type III fracture. There were 34 patients with fracture occurred due to accidental fall and in 10 patients it was due to RTA. Out of the 44 patients, 24 patients didn't have any comorbidities, 6 patients had only HTN, 10 patients had both DM/HTN and 4 patients had only DM. Radiological union was said to be achieved on the evidence of obliteration of fracture lines and trabecular continuity between the two fragments on AP \& lateral x-rays in three cortices. In 31 patients fracture is united between 10-12 weeks, in 12 patients united between13-15 weeks, and in 1 patients union took more than 15 weeks. Mean union time was 12.02 weeks.

In $55 \%$ of patients surgery was done with-in one day after injury. The mean duration of interval between injury and surgery was 1.72 days.

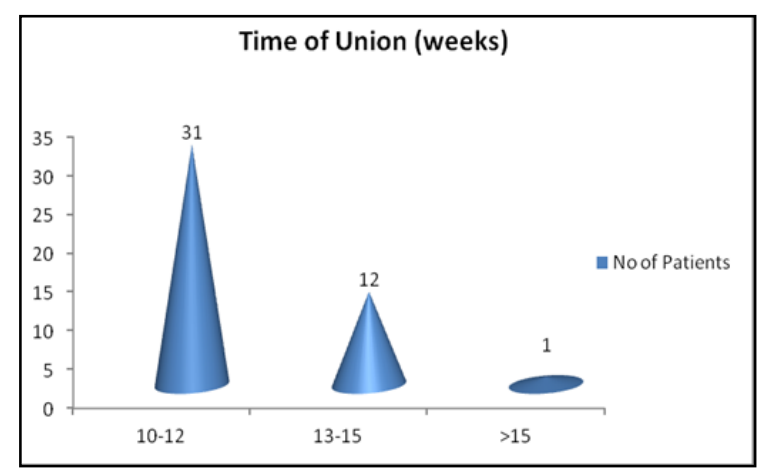

Graph 3: Time of union

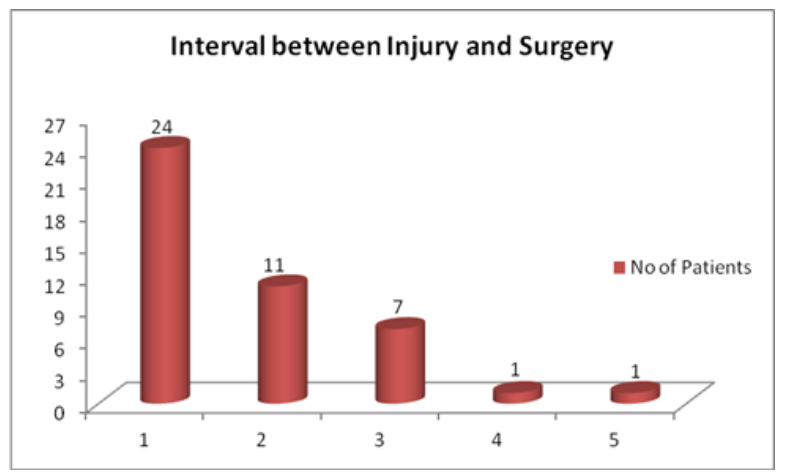

Graph 4: Interval between injury and surgery 
In $75 \%$ of patients duration of hospital stay was between 4-7 days. $20 \%$ stayed more than 8 days. The mean duration of hospital stay was 6.15 days. In $46 \%$ of the patients operating time was $61-75$ minutes, in $11 \%$ patients operating time was more than 75 minutes and in $43 \%$ patients it was less than 60 minutes. Average operating time was 65.59 minutes. In the study 8 patients had $150 \mathrm{ml}$ of blood loss, 13 patients had 200 $\mathrm{ml}, 19$ patients had $250 \mathrm{ml}$, and 4 patients had $300 \mathrm{ml}$. Average blood loss in the study population is $221.59 \mathrm{ml}$.
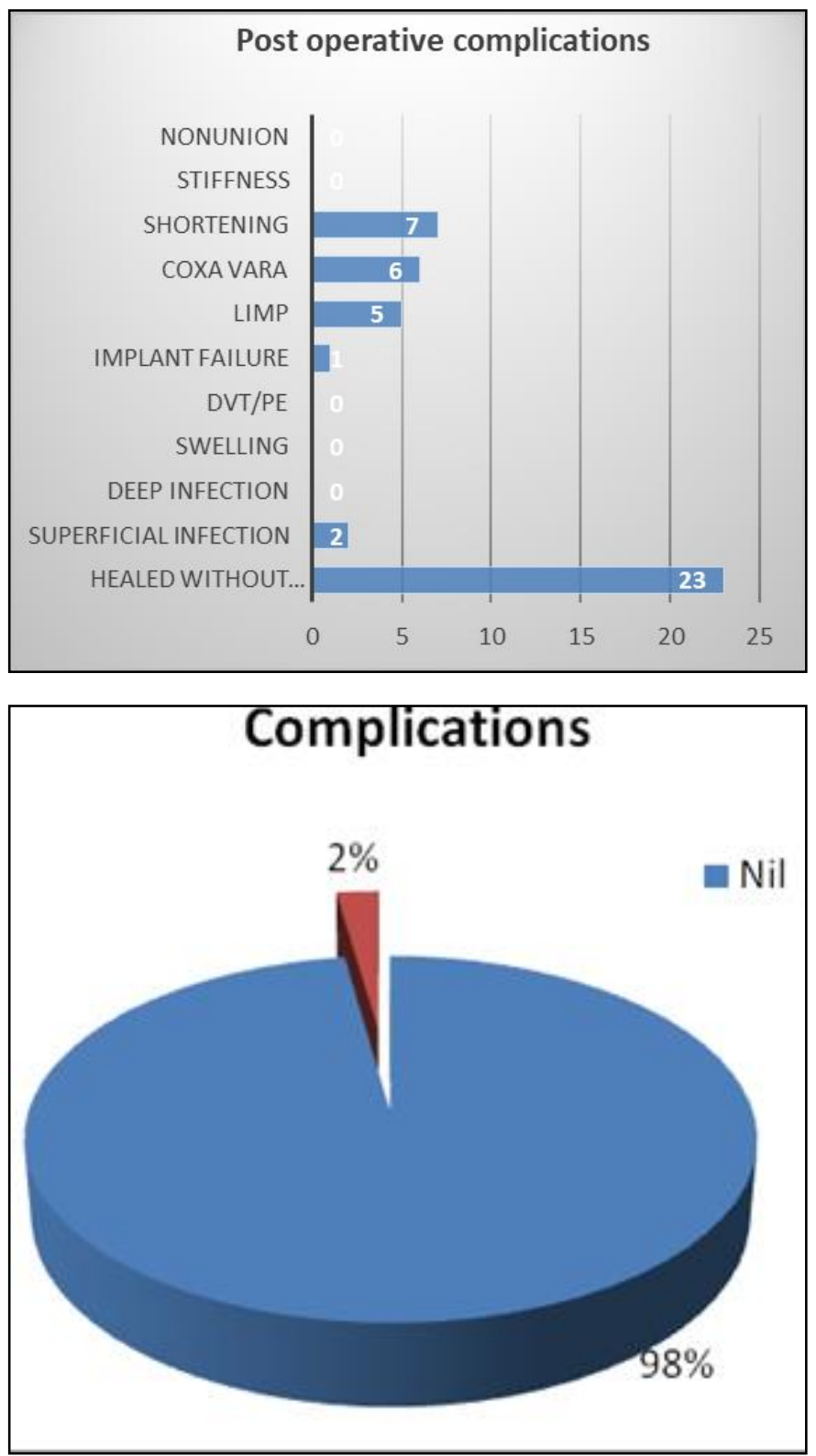

Graph 5: Post-operative and Implant related complications in the study population

In the study, 23 patients healed well without any postoperative complications. 2 patients developed superficial infection which healed well with i.v. antibiotics for 3 weeks. Limping was present in 5 cases, coxa vara in 6 cases, shortening in 7 cases and implant failure in 1 patient. One patient $(2 \%)$ in the study population, developed complications related to the implant, that is helical blade cut-out. In the study, $36 \%$ patients had $21-25,39 \%$ patients had $26-30$, and $2 \%$ patients had TAD more than 30 and mean TAD was 23.95 .

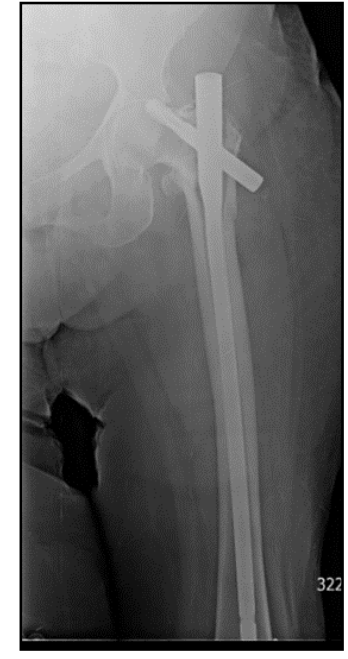

Fig 2: Helical blade cut out

In this study, $37(84 \%)$ patients had excellent and good outcome and 7 (16\%) had fair and poor outcome.

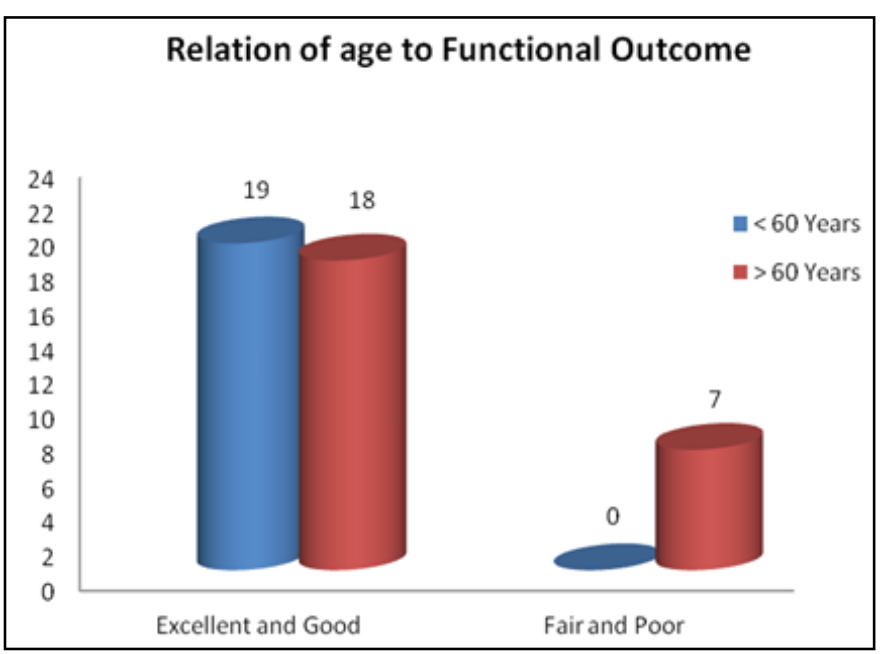

Graph 6: Relation of age to functional outcome

Relation of Co-morbodities to functional Outcome

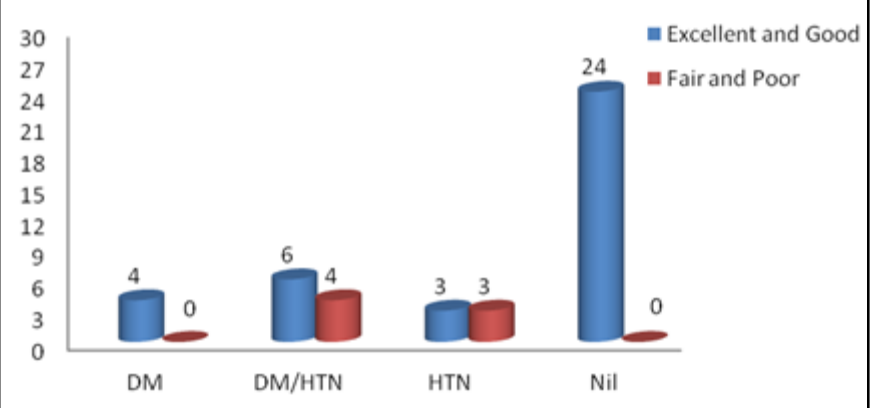

Graph 7: Relation of comorbidities to the outcome

In the study, 19 patients were less than 60 years of age group and all patients of this group had excellent and good outcome, 25 patients were belongs to more than 60 years of age group of which 7 patients had fair and poor outcome and it is statistically significant $(\mathrm{p}=0.0139)$. 
Out of the 44 patients, 24 patients didn't have any comorbidities, of which all had excellent and good outcome.6 patients had only HTN, of which 3 had excellent and good outcome and 3 patients had fair and poor outcome. 10

patients had both DM/HTN of which 6 had excellent and good outcome and 4 patients had fair and poor outcome. 4 patients had only DM, of which all had excellent and good outcome. Comorbidities are statistically significant association with outcome ( $\mathrm{p}=0.002)$.

In this study, 23(52\%) patients had type II fracture of which 18 patients had excellent and good out-come and 5 patients had fair and poor out-come. Eleven patients had type I fracture of which 9 patients had excellent and good out-come and 2 patients had fair and poor out-come. 10 patients had type III fracture of which all had excellent and good outcome. We found no statistically significant relation between type of fracture and functional out-come $(\mathrm{p}=0.283)$.

In this study 26 patients had TAD less than 25 of which nobody had implant related complications, and 18 patients had TAD more than 25 of which one patient had implant related complications. We found no statistically significant relation between TAD values and implant related complications. $(\mathrm{p}=0.22)$

\section{Discussion}

Many authors have investigated the functional outcomes after treating intertrochanteric fractures by PFNA in order to achieve rapid pre-operative status and early union with less complication. Others have sought to identify the role of certain implant fixation which predict the failure of proximal femoral nail antirotation.

The mean age of the study population was 65.02 years. Mean age of the different studies published in literature had wide range of distribution. In Ming Hui Li et al. ${ }^{[4]}$ study, total numbers of patients were 163 with mean age of 74.7 years. In Jin-Song Pu et al. ${ }^{[5]}$ study, total number of patients were 87 with mean age of 75.3 years.

In our study, male to female ratio was 25:19, while this ratio was 69:94 in Ming Hui Li et al. ${ }^{[4]}$, while this ratio was 21:66 in Jin-Song $\mathrm{Pu}$ et al. ${ }^{[5]}$ study.

In our study, mode of injury (accidental fall to RTA) ratio was 34:10, while this ratio was 117:46 in Ming Hui Li et al. ${ }^{[4]}$ study, and 63:24 in Jin-Song Pu et al. ${ }^{[5]}$ study.

In our study, we found that mean age of the patients who had Excellent and Good outcome was lower than mean age of the patients with fair and poor outcome $(p=0.0139)$. Hence, we conclude that age is a significant factor contributing to final outcome. Other studies in the literature also observed similar findings where age was a predictor of final outcome.

In our study, 20 patients had co-morbid conditions. 7 out of 20 had fair and poor outcome $(\mathrm{p}=0.002)$. Hence, we conclude that co-morbid conditions significantly affect

Functional outcome after hip surgery. Radosavljevic $\mathrm{N}$ et al. [5] in 2013 studied 203 patients with hip fractures and concluded that male sex, increased co-morbid conditions and age more than 85 years could be considered to have lower functional outcome potential after hip surgery.

In our study, 7 patients had fair and poor functional outcomes of which $5(71 \%)$ patients had type II fracture. 2 (29\%) patients had type I fracture. We did not find a

Significant association between type of fracture and functional out-come $(p=0.283)$. Hence we conclude that type of fracture is not a significant factor contributing to final outcome.

Most common postoperative implant related complication encountered during radiological evaluation is proximal cut out, with incidence of range 0.7 to $10.6 \%{ }^{[7]}$. In

Simmermacher RK et al. ${ }^{[8]}$ study, $3.6 \%$ patients had proximal blade cut-out this is comparable to our study. In MingHui Li et al. ${ }^{[4]}$ study, and in Jin-Song Pu et al. ${ }^{[5]}$ study no cut-outs were seen. In our study, one patient $(2 \%)$ had blade cut-out. Cut-out patient TAD was $30 \mathrm{~mm}$ (more than $25 \mathrm{~mm}$ ), but TAD was statistically insignificant factor to implant related complications in our study. This patient fracture was united in varus position and re-operated at 4 months for blade removal. In our study, 23(52.2\%) patients healed well without any post-operative complications. Two $(4.5 \%)$ patients developed superficial infection which healed well with i.v. antibiotics for 3 weeks and none of our patients had deep infection.

Limp: In our study, $88 \%$ of the patients had a normal gait by one year. 5 patients in our study had a limp on the affected side.

Coxa vara: In our study, $87 \%$ of the patients had a neck-shaft angle of $130^{\circ}$ or more. 2 patient had varus angulation of $5^{\circ}, 3$ had varus angulation of $10^{\circ}$ and 1 patients had varus angulation of $15^{\circ}$. No case had varus angulation of more than $15^{\circ}$.

Varus angulation of up to $15^{\circ}$ is acceptable and does not need re-doing.

Shortening: In our study, $85 \%$ of our patients had no shortening. 4 patients developed shortening of $1 \mathrm{~cm}$ and 3 patients developed shortening of $2 \mathrm{~cm}$. No case had shortening of $3 \mathrm{~cm}$ or more.

In our study, Singh's osteoporosis index ${ }^{[9]}$ was not considered during the management of intertrochanteric fractures. This is because other than Singh's osteoporosis index many other significant implant fixation factors which predict the failure of proximal femoral nail antirotation have been reported in literature. Kashigar A et al. in 2014, retrospectively reviewed 77 proximal femoral fractures with a objective of determining the factors predicting the failure of cephalomedullary nailing and observed that non-significant parameters were age, gender, fracture type, Singh's osteoporosis index, reduction quality and significant parameters were TAD, Cal TAD and cervical angle difference.

No cases of AVN (Avascular necrosis) of femoral head and non-union were noted in our study.

The general incidence of mortality after trochanteric fractures is described in the current literature as 6 to $11 \%$ within first month and 14 to $36 \%$ within the first year.

Mortally typically results from from cardiopulmonary, thromboembolic and septic complications. In our study, the mortality rate was $4 \%$ ( 2 patients) over the one-year period. The cause of death was bronchopneumonia in both the cases. In our study, $84 \%$ patients had excellent and good outcome and $16 \%$ patients had fair and poor outcome at the end of one year follow-up. This is comparable with other studies in the literature. In MingHui Li et al. ${ }^{[4]}$ study, $81.57 \%$ had excellent and good outcome and $18.43 \%$ had fair and poor outcome. In Jin-Song $\mathrm{Pu}$ et al. ${ }^{[5]}$ study, $77 \%$ had excellent and good outcome and $23 \%$ had fair and poor outcome. In WL Loo et al. ${ }^{[10]}$ study, $83.9 \%$ had excellent and good outcome and $16.1 \%$ had fair and poor outcome.

Our study shows $84 \%$ favorable functional outcome but $16 \%$ of the patients suffered from the known complications and did not returned to their pre fracture mobility status.

A larger sample size would be required to extrapolate the 
result on to the general population. Preoperative walking ability of the patients was not determined which may affect the final functional status achieved. Osteoporotic nature of the proximal femur is not considered which may affect the implant fixation and complications like blade cut-out, but recent studies are against this.

\section{Conclusions}

Peritrochanteric fractures especially in the elderly patients are significant life event and patients as well as their family members should be counselled about the potential problems and their expectations should be set appropriately. Closed reduction and internal fixation is an excellent method of treatment for an intertrochanteric fracture, to provide rapid recovery and good functional outcome. Age is a significant predictor of final outcome following surgical treatment. Younger is the age better the outcome.

Gender, laterality and mode of injury, Type of injury are not a predictor of final outcome. Co-morbid conditions mainly diabetes, hypertension and complications related to surgical treatment are significant predictors of final outcome. Especially in unstable intertrochanteric fractures PFNA offers the advantages of less blood loss, shorter operative time and reduced re-operative rate when compared with commonly used stabilization systems such as DHS.

\section{References}

1. Spears GN, Owen JT. The etiology of trochanteric fractures of the femur. J Bone Joint Surg Am. 1949; 31(3):548-52.

2. Kannus P, Parkkari J, Sievänen H, Heinonen A, Vuori I, Järvinen M. Epidemiology of hip fractures. Bone. 1996; 18(1):57-63.

3. Koval KJ, Aharonoff GB, Rokito AS, Lyon T, Zuckerman JD. Patients with femoral neck and intertrochanteric fractures: Are they the same? Clinical orthopaedics and related research. 1996; 330:166-72.

4. Li M, Wu L, Liu Y, Wang C. Clinical evaluation of the Asian proximal femur intramedullary nail antirotation system (PFNA-II) for treatment of intertrochanteric fractures. Journal of orthopaedic surgery and research. 2014; 9(1):1.

5. Pu JS, Liu L, Wang GL, Fang Y, Yang TF. Results of the proximal femoral nail anti-rotation (PFNA) in elderly Chinese patients. International orthopaedics. 2009; 33(5):1441-4.

6. Radosavljevic N, Nikolic D, Lazovic M, Petronic I, Milicevic V, Radosavljevic $\mathrm{Z}$ et al. Estimation of functional recovery in patients after hip fracture by Berg Balance Scale regarding the sex, age and comorbidity of participants. Geriatrics \& gerontology international. 2013; 13(2):365-71.

7. Kaplan K, Miyamoto R, Levine BR, Egol KA, Zuckerman JD. Surgical Management of Hip Fractures: An Evidence- based Review of the Literature. II: Intertrochanteric Fractures. Journal of the American Academy of Orthopaedic Surgeons. 2008; 16(11):665-73.

8. Simmermacher RK, Ljungqvist J, Bail H, Hockertz T, Vochteloo AJ, Ochs U. The new proximal femoral nail antirotation (PFNA®) in daily practice: results of a multicentre clinical study. Injury. 2008; 39(8):932-9.

9. Singh M, Nagrath A, Maini PS. Changes in trabecular pattern of the upper end of the femur as an index of osteoporosis. J Bone Joint Surg Am. 1970; 52(3):457-67.
Antirotation (PFNA) and PFNA-2-Our Local Experience. Malaysian Orthopaedic Journal. 2011; $5(2): 1-5$

10. Loo WL, Loh SY, Lee HC. Review of Proximal Nail 